\title{
Neue Strategie bei PDT: Gleicher Erfolg, weniger Nebenwirkungen
}

\author{
Eine verkürzte Einwirkzeit des Photosensibilisators und die zusätzliche Applikation eines lokalen \\ Kortikoids können einer dänischen Studie zufolge dazu beitragen, das Nebenwirkungspotenzial \\ der photodynamischen Therapie bei gleicher Effizienz zu verringern. Dies könnte dazu führen, \\ dass die PDT bei der größerflächigen Behandlung der aktinischen Keratose an exponierten \\ Stellen künftig mehr Akzeptanz erfährt.
}

Die stärksten Nebenwirkungen einer photodynamischen Therapie (PDT) zur Behandlung einer aktinischen Keratose (AK) sind Schmerzen während der Bestrahlung, posttherapeutische Ödeme sowie Hautrötungen und Entzündungserscheinungen. Diese weithin sichtbaren Begleiterscheinungen legen Patienten, die in der Öffentlichkeit stehen, oft über Wochen lahm. Aber auch viele weniger exponierte Menschen lassen sich nicht gerne mit den Entzündungen und Verkrustungen sehen. Entsprechend schlecht ist die Akzeptanz solcher an sich wirksamen Therapieformen, wenn die Behandlung etwa im Gesicht oder am Kopf über große Flächen erforderlich ist.

Im Rahmen einer randomisierten intraindividuellen Studie haben Stine Wiegell und Kollegen vom Bispebjerg Hospital in Kopenhagen jetzt nach Möglichkeiten gesucht, mit veränder-

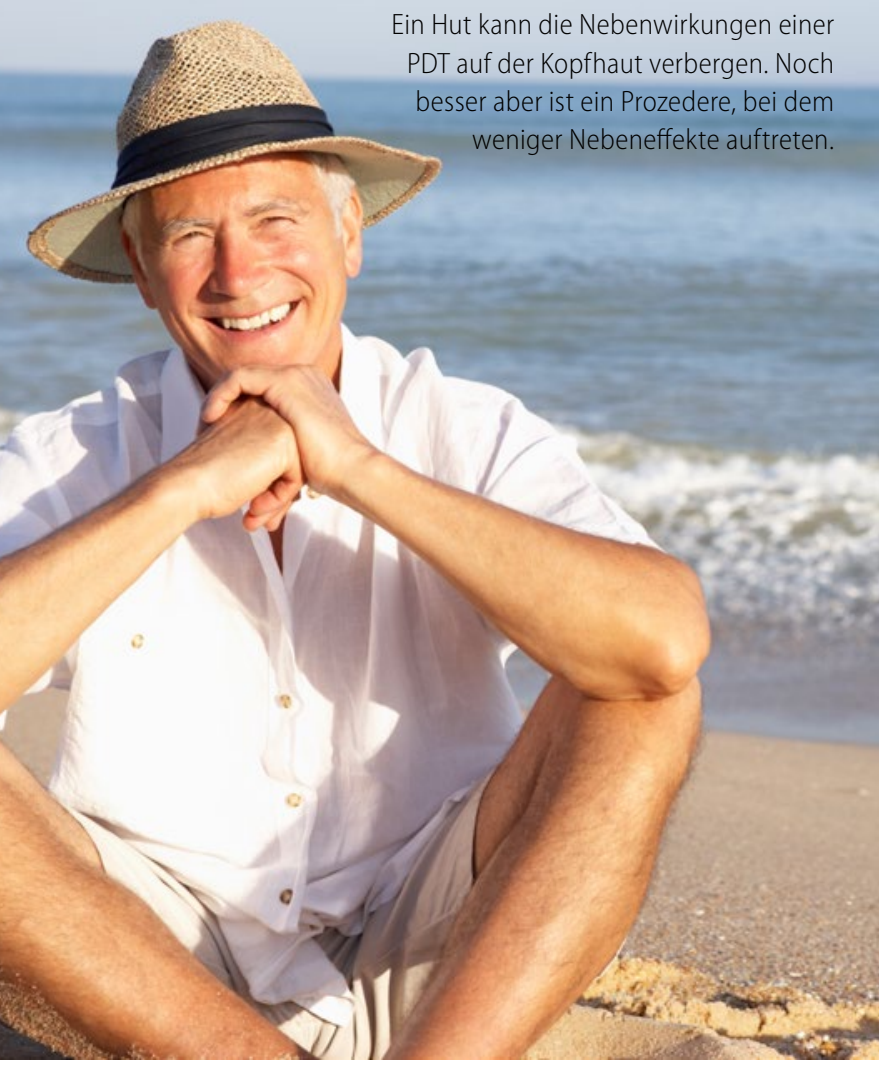

tem Prozedere bei gleicher Wirksamkeit weniger Nebeneffekte zu erzielen als mit der konventionellen Methode (PDT mit Methylaminolävulinat, MAL, für drei Stunden). Die Dermatologen verkürzten die Einwirkzeiten des Photosensibilisators auf 30 Minuten (Pulse-PDT) bzw. behandelten die zu bestrahlenden Hautstellen vor und direkt nach der Pulse-PDT zusätzlich mit einer hochpotenten Kortikoidsalbe (S-Pulse-PDT). Eine gewisse Inkubationszeit ist erforderlich, damit die Porphyrinvorstufe MAL in die Keratinozyten aufgenommen und dort über den Häm-Biosyntheseweg zum photosensitiven Protoporphyrin IX (PpIX) umgewandelt werden kann. Durch die Kortikoidbehandlung kommt es vor der Bestrahlung zu einer signifikant stärkeren PpIX-Fluoreszenz als ohne diese zusätzliche Behandlung.

21 immunkompetente Männer und eine Frau mit leichten Formen multipler aktinischer Keratosen im Gesicht und auf der Kopfhaut wurden in jeweils drei ähnlichen Regionen entsprechend unterschiedlich behandelt. Nach drei Stunden wurden alle Regionen der durchschnittlich 76-Jährigen mit Rotlicht bestrahlt. Die Hautrötung wurde in Vorbereitung auf die Bestrahlung sowie am darauffolgenden Tag mit einem Reflektometer bestimmt. AK-Läsionen in allen Therapiebereichen wurden gezählt, eingestuft und dokumentiert. Drei Monate nach der Behandlung wurden die behandelten Gebiete mit dem Ausgangsbefund verglichen.

\section{Beste Ergebnisse bei zusätzlicher Kortikoidbehandlung}

An den Pulse-PDT-behandelten Stellen ergab sich 24 Stunden nach der Therapie insgesamt eine signifikant schwächere Rötung als nach konventioneller PDT. Noch weniger Entzündungserscheinungen zeigten sich, wenn zusätzlich Kortisonsalbe appliziert worden war (S-Pulse-PDT). Während sich bei konventionell behandelten Patienten immer eine Rötung ergab, waren bei einem Patienten der Pulse-PDT- und vier Patienten der S-Pulse-PDT-Gruppe keinerlei Erytheme erkennbar. Hellrot mit Verhärtungen zeigten sich die Hauterscheinungen dagegen bei neun Patienten der PDT-Gruppe, bei nur drei der PulsePDT- und bei keinem der S-Pulse-PDT-Patienten. Die Behandlungserfolge nach drei Monaten unterschieden sich zwischen den drei Therapiearten nicht signifikant.

Dr. Christine Starostzik 